\title{
Chandra observations of the X-ray emission from Molecular Clouds at the Galactic Center related to Sgr $A^{\star}$ past activity
}

\author{
Maïca Clavel $^{* a, b}$, R. Terrier ${ }^{a}$, A. Goldwurm ${ }^{a, b}$, M. R. Morris ${ }^{c}$, G. Ponti ${ }^{d}$, S. Soldi ${ }^{a}$, \\ G. $\operatorname{Trap}^{e, a, b}$ \\ ${ }^{a}$ AstroParticule et Cosmologie, Univ. Paris Diderot, CNRS/IN2P3, CEA/DSM, Observatoire de \\ Paris, Sorbonne Paris Cité ; 10, rue A. Domon et L. Duquet, 75205 Paris Cedex 13, France \\ ${ }^{b}$ Service d'Astrophysique/IRFU/DSM, CEA Saclay, 91191 Gif-sur-Yvette Cedex, France \\ ${ }^{c}$ Dept. of Physics \& Astronomy, University of California, Los Angeles, CA 90095-1547, USA \\ ${ }^{d}$ Max-Planck-Institute for Extraterrestrial Physics, PSF 1312, D-85741 Garching, Germany \\ ${ }^{e}$ Unité de physique, Palais de la découverte - Universcience, 75008 Paris, France \\ E-mail: maica.claveleapc.univ-paris7.fr
}

\begin{abstract}
The relatively rapid spatial and temporal variability of the X-ray radiation from some molecular clouds near the Galactic center shows that this emission component is due to the reflection of $\mathrm{X}$-rays generated by a source that was very luminous in the past, most likely the central supermassive black hole, Sagittarius $A^{\star}$. Studying the evolution of the molecular cloud reflection features is therefore a key element to reconstruct $\mathrm{Sgr} \mathrm{A}^{\star}$ 's past activity. The aim of our work was to study this emission on small angular scales in order to measure the reflected emission on short time scales and thereby to highlight the similarities in the cloud temporal behaviors. We used Chandra high-resolution data collected from 1999 to 2011 to study the variations of emission within clouds between $5^{\prime}$ and $20^{\prime}$ from Sgr $A^{\star}$ towards positive longitudes. The analysis of the reflection emission in the 4-8 keV energy range allowed us to perform a systematic characterization of the variations down to $15^{\prime \prime}$ angular scale and one-year time scale. In particular, it reveals that five molecular clouds in the region of interest show significant variations in which we identify two distinct behaviors: a short peaked emission propagating along two of the clouds and slower linear variations for the other three molecular clouds. We believe the illumination of the region is due to the reflection of at least two distinct past outbursts of $\operatorname{Sgr} A^{\star}$.
\end{abstract}

An INTEGRAL view of the high-energy sky (the first 10 years) - 9th INTEGRAL Workshop and celebration of the 10th anniversary of the launch

15-19 October 2012

Bibliothèque Nationale de France, Paris, France

* Speaker. 


\section{Introduction}

$\operatorname{Sgr~A}^{\star}$ is the supermassive black hole located in the center of the Galaxy. Its quiescent X-ray luminosity is about $10^{33-34} \mathrm{erg} \mathrm{s}^{-1}$ [1] but varies, showing rapid flares [2] during which its luminosity remains at least eight orders of magnitude lower than its Eddington luminosity. The recent detection of a dense gas cloud falling towards the accretion zone of Sgr $\mathrm{A}^{\star}$ [3] provides a hint that the accretion rate onto Sgr $\mathrm{A}^{\star}$ may vary and since Active Galactic Nuclei (AGN) have a short duty cycle $\left(\sim 10^{-2}\right)$ [4], Sgr $\mathrm{A}^{\star}$ is compatible with being an AGN in a temporary low state.

There are strong hints that $\mathrm{Sgr} \mathrm{A}^{\star}$ experienced a large phase of activity in the past [5] and its recent history can be reconstructed from the non-thermal emission emanating from the molecular clouds at the Galactic center. This emission is characterized by a continuum component and a strong $\mathrm{Fe} \mathrm{K} \alpha$ line at $6.4 \mathrm{keV}$. The strong variability of this emission, detected in both Sgr B2 $[6,7,8]$ and the Sgr A region $[9,10,11,12]$, proves that a significant fraction of the diffuse emission correlated with the molecular clouds is due to reflection. This reflected emission is created by Compton scattering and K-shell photo-ionization of neutral iron atoms produced by intense X-ray radiation such as emitted during a putative past large flare of $\operatorname{Sgr} A^{\star}[13,14,15]$. Unfortunately, reconstructing the lightcurve of $\operatorname{Sgr} \mathrm{A}^{\star}$ is very complex because the distribution of clouds along the line of sight is barely known. Since the illuminated temporal behavior of clouds is directly linked to their relative position along the line of sight, characterizing the fine $\mathrm{X}$-ray variations is essential for understanding the past activity of Sgr $\mathrm{A}^{\star}$.

We took advantage of Chandra's high spatial resolution to identify the fine variations occurring in a key region located between $\mathrm{Sgr}^{\star}$ and the radio arc [12]. This region, hereafter called the Sgr A complex, is composed of five main molecular clouds: MC1, MC2, Br1, Br2 and G0.110.11, all identified in Fig. 1. Here, we present the main results concerning the temporal variations of these clouds. Our analysis is based on all the Chandra observations covering the ten arcmin squared region centred on $(1, b)=\left(0.06^{\circ}, 0.10^{\circ}\right)$, that have been collected between 1999 and 2011. The data have been reduced using Chandra standard analysis as described in Clavel et al. [12], and we use the 4-8 keV flux of 15 arcsec squared regions in order to characterize the variations of the Sgr A complex. This systematic analysis of the region is in perfect agreement with the $6.4 \mathrm{keV}$ characterization also performed for some specific clouds in Clavel et al. [12] and shows that all five molecular clouds are varying with a significance of at least $10 \sigma$ but with two different types of behavior: the variations are either short (year) and peaked or slower (decade) and linear.

\section{Two-year peaked variations in the Br1 and Br2 clouds}

$\mathrm{Br} 1$ and $\mathrm{Br} 2$ are two molecular clouds appearing as a coherent structure in molecular surveys of the region $[16,17]$. In the X-rays, this structure, called the Bridge, has mainly been illuminated since 2008 (Fig. 1), the illumination started on the Galactic west of the cloud with a superluminal propagation of the echo away from $\mathrm{Sgr}^{\star}{ }^{\star}[10]$. Our analysis confirms the propagation along the Bridge, with the detection of a bright filament at the center of $\mathrm{Br} 2$ in 2011 (Fig. 1, lightcurve 2). This sharp increase seen in the eastern part of the Bridge is fully compatible with the increase displayed by several other filaments in Br1, which is again an indication of a rather contiguous and coherent molecular structure witnessing the same illuminating event. 

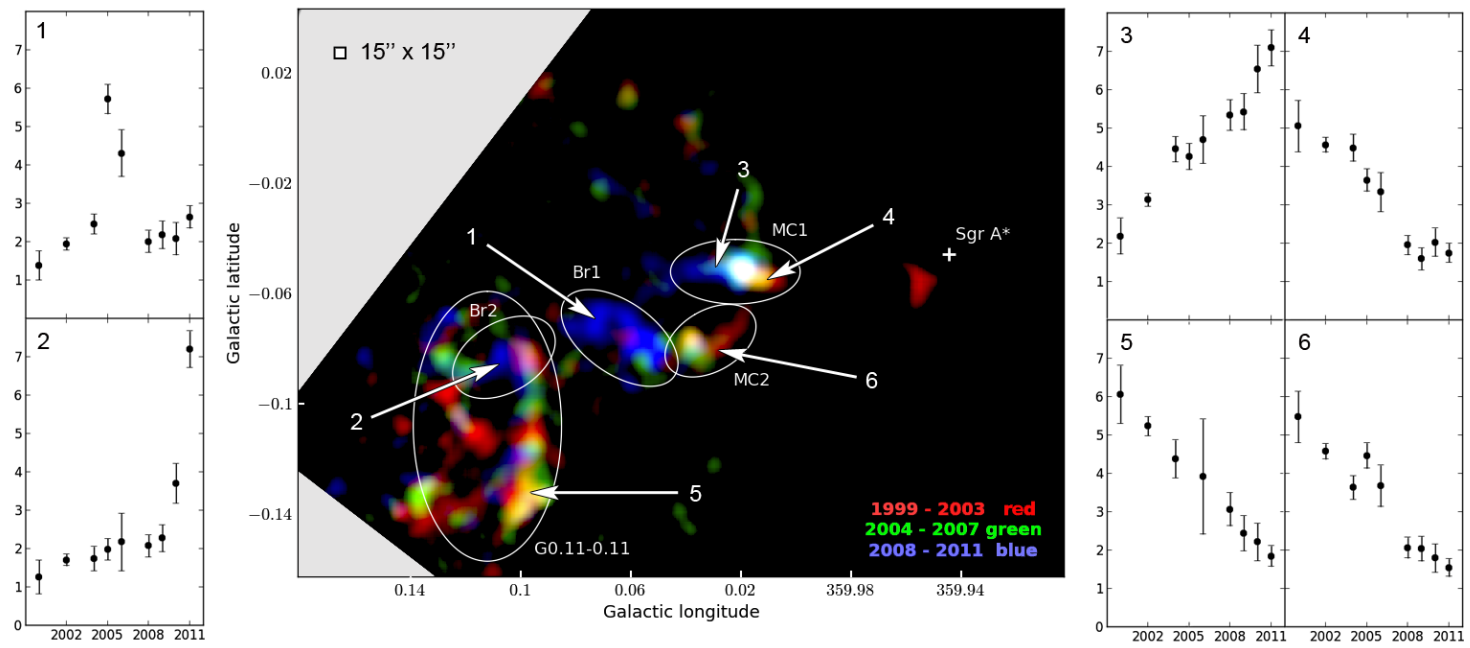

Figure 1: Center: Continuum subtracted three-color image of the $\mathrm{Fe} \mathrm{K} \alpha$ emission in the Galactic center region as seen by Chandra from 1999 to 2003 (red), 2004 to 2007 (green), 2008 to 2011 (blue). The images are smoothed with a gaussian kernel of 9 arcsec. The image highlights an apparent propagation of the emission, moving away from Sgr $\mathrm{A}^{\star}$. Left and Right: $4-8 \mathrm{keV}$ flux lightcurves in units of $10^{-6} \mathrm{ph} \mathrm{cm}^{-2} \mathrm{~s}^{-1}$ extracted on 15 arcsec squared regions whose positions are indicated by arrows on the central image. Linear variations on 10-year time scales are seen in all clouds except for $\mathrm{Br} 1$ and $\mathrm{Br} 2$, which are experiencing faster variations.

Moreover, the systematic fit of the lightcurves for 15-arcsec-squared regions provides at least a $5 \sigma$ rejection of both the constant and linear models for most of the Bridge subregions. In particular, we report the first detection of a flux increase followed by a similar decrease in several subregions of the Br1 cloud (Fig. 1, lightcurve 1), which fixes the duration of the reflection episode to about two years. Since the reflected lightcurve variation cannot be faster than the illuminating event itself, this new measurement implies that the past X-ray radiation responsible for the $\mathrm{Br} 1$ and $\mathrm{Br} 2$ illumination peak lasted no more than two years.

By reporting such a short event, we highlight the importance of small-scale characterization and call into question few-arcmin-scale analyses, which are partly hiding these fast and fine variations by averaging them.

\section{Ten-year linear variations in MC1, MC2 and G0.11-0.11 clouds}

MC1, MC2 and G0.11-0.11 are also three coherent molecular structures [16, 17]. Our smallscale analysis of $\mathrm{MC} 1$ reveals that its emission is not constant as has been previously reported $[10,11]$, but shows morphology changes on a few-year time scale. The variation is characterized by a ten-year linear increase in the eastern part of the cloud (Fig. 1, lightcurve 3) and a ten-year linear decrease in the western part of the cloud (Fig. 1, lightcurve 4). Once again, the small-scale analysis we performed reveals variations that are more complex than previously anticipated by larger scale analyses.

Our analysis of the MC2 and G0.11-0.11 clouds shows that they have undergone a linear flux decrease for the past ten years (Fig. 1, lightcurves 5 and 6). The slopes of the linear decreases 
seen in all three clouds are very similar. This is a strong indication that this variation pattern is mainly dominated by the time scale of the illuminating event rather than by each cloud's specific configuration. Therefore, these three structures are likely to be seeing the same event almost simultaneously. This gives strong constraints on their relative positions along the line of sight but since we do not know the timing of the illuminating event their absolute position cannot be assessed.

\section{Discussion and Conclusions}

Our systematic analysis of the 4-8 keV flux variations of the Sgr A complex, divided into 15 arcsec squared subregions, reveals significant variations in all the molecular clouds of the complex. We report two different types of behavior: on one hand a two-year peaked variation for the $\mathrm{Br} 1$ and $\mathrm{Br} 2$ clouds (Section 2) which is the fastest variation detected so far in the diffuse X-rays from Galactic center molecular clouds, and on the other hand a ten-year linear variation for the other three clouds (Section 3), with morphology changes visible on few-arcsec scales.

As discussed in Clavel et al. [12], the relative densities of the clouds as well as the similarity of the decreasing trend observed in the three clouds having ten-year variations suggest that the two different behaviors are due to two distinct illuminating events. Having an estimate of the events' luminosity is difficult since both the position of the cloud along the line of sight and the opacity of the clouds on scales that match the X-ray data are unknown. Nevertheless, assuming reasonable parameters for the clouds and a source at the position of Sgr $\mathrm{A}^{\star}$ we find that two flares of few $10^{39} \mathrm{erg} \mathrm{s}^{-1}$ lasting for two and ten years are sufficient to explain the short and long behaviors, respectively.

This two-flare scenario questions $\mathrm{Sgr} \mathrm{A}^{\star}$ as the privileged candidate and we investigated whether a Galactic X-ray transient could account for the echoes [12], and in particular for the short behavior seen in the Bridge. Two plausible alternative origins are X-ray binaries and Soft Gamma Repeaters (SGR). However, only a peculiar binary having a burst of one year with a luminosity similar to that of GRS $1915+105$ could explain the short behavior if located twice closer to the cloud than the minimal distance of $\mathrm{Sgr} \mathrm{A}^{\star}$, while outbursts from typical X-ray binaries [18] are excluded. Due to the recent discovery of SGR J1745-29 located only 3" from Sgr A* [19], the possibility that a SGR giant flare is at the origin of the molecular cloud illumination has also been carefully considered [12]. We have shown that even the most powerful event of the three detected so far, the one of SGR 1806-20 in 2004 [20], but emitted by SGR J1745-29 at the Galactic center cannot explain what is observed. Therefore, even if we cannot exclude more extreme events from transient sources, Sgr $\mathrm{A}^{\star}$ is the preferred explanation for the two outbursts reflected by the molecular clouds of the Sgr A complex.

The discovery of both the short illuminating event and the morphology changes on few-arcsec scales highlights the importance of small-scale characterization and proves that even if large-scale analyses give better statistics, they also average over the intrinsic cloud behavior and therefore they may hide important information. This is why some of our results are apparently inconsistent with larger scale analyses and suggest an activity period of $\mathrm{Sgr} \mathrm{A}^{\star}$ with much faster variations than what was proposed in previous works $[10,11]$. Our results provide new and significant constraints for the identification of the physical processes responsible for the past changes in the luminosity of Sgr $A^{\star}$. 


\section{Acknowledgments}

The authors acknowledge support from the International Space Science Institute to the International Team 216 and financial support from the UnivEarthS Labex program of Sorbonne Paris Cité (ANR-10-LABX-0023 and ANR-11-IDEX-0005-02). The scientific results reported in this article are based on observations made by the Chandra X-ray Observatory and on data obtained from the Chandra Data Archive. MC acknowledges the Université Paris Sud 11 for financial support. MM acknowledges support from NASA. GP acknowledges support via an EU Marie Curie Intra-European fellowship under contract no. FP-PEOPLE-2012-IEF-331095. SS acknowledges the Centre National d'Etudes Spatiales (CNES) for financial support.

\section{References}

[1] Baganoff F. K., Maeda Y., Morris M. et al. 2003, ApJ, 591, 891

[2] Nowak M. A., Neilsen J., Markoff S. B. et al. 2012, ApJ, 759, 95

[3] Gillessen S., Genzel R., Fritz T. K. et al. 2012, Nature, 481, 51

[4] Greene J. E. \& Ho L. C. 2007, ApJ, 667, 131

[5] Ponti G., Morris M. R., Terrier R. et al. 2013, in Advances in Solid State Physics, Vol. 34, Cosmic Rays in Star-Forming Environments, ed. D. F. Torres \& O. Reimer, 331

[6] Inui T., Koyama K., Matsumoto H. et al. 2009, PASJ, 61, 241

[7] Terrier R., Ponti G., Bélanger G., et al. 2010, ApJ, 719, 143

[8] Nobukawa M., Ryu S. G., Tsuru T. G., et al. 2011, ApJL, 739, L52

[9] Muno M. P., Baganoff F. K., Brandt W. N., et al. 2007, ApJL, 656, L69

[10] Ponti G., Terrier R., Goldwurm A. et al. 2010, ApJ, 714, 732

[11] Capelli R., Warwick R. S., Porquet D. et al. 2012, A\&A, 545, A35

[12] Clavel M., Terrier R., Goldwurm A. et al. 2013, A\&A, in Press, [ArXiv:1307.3954]

[13] Sunyaev R. A., Markevitch M. \& Pavlinsky M. 1993, ApJ, 407, 606

[14] Koyama K., Maeda Y., Sonobe T. et al. 1996, PASJ, 48, 249

[15] Sunyaev R. A. \& Churazov E. 1998, MNRAS, 420, 388

[16] Tsuboi M., Handa T. \& Ukita N. 1999, ApJS, 120, 1

[17] Jones P. A., Burton M. G., Cunningham M. R. et al. 2012, MNRAS, 419, 2961

[18] Dunn R. J. H., Fender R. P., Körding E. G., et al. 2010, MNRAS, 403, 6

[19] Kennea, J. A., Burrows, D. N., Kouveliotou, C., et al. 2013, ApJL, 770, L24

[20] Hurley K., Boggs S. E., Smith D. M., et al. 2005, Nature, 434, 1098 